\title{
Correction for guessing in choice reaction time
}

\author{
JOHN I, YELLOTT, JR, \\ UNIVERSITY OF MINNESOTA
}

Additional theoretical and experimental results are presented for a choice reaction time performance model described by Ollman (1966). A formula is given for estimating the latency distribution of true recognition responses from the results of a single session; the estimate is invariant with respect to changes in the proportion of "guess" responses and with respect to fluctuations in the latency distribution of guesses.

A recent note by Ollman (1966) describes a model for performance in choice reaction time (CRT) situations. According to this model the $S$ in a CRT experiment makes two kinds of responses: fast "guess" responses, and slower responses which represent the outcome of a (possibly imperfect) recognition process. The latter will be referred to as "stimulus controlled responses" (SCRs). The relative frequency of guesses and SCRs is controlled by the $S$, and presumably reflects his effort to attain an optimal balance between speed and accuracy. Ollman presents results which allow the parameters of the model to be estimated if data are available from several experimental sessions-corresponding to several different observed proportions of correct and incorrect responses. The present note reports an additional result which allows the "true" reaction time distribution, i.e., the latency distribution of the stimulus controlled responses, to be estimated from the data of a single session. The equation expressing this result can be regarded as a "correction for guessing" formula. Further experimental tests of the model are also reported.

Consider a CRT task involving two stimuli $S_{1}$ and $S_{2}$. The subject is instructed to make response $A_{i}(i=$ 1 ,2) when $S_{1}$ is presented, and we assume that $S_{1}$ and $\mathrm{S}_{2}$ are presented randomly over trials with $\mathrm{P}\left(\mathrm{S}_{1}\right)=.5$. The model supposes that on any trial the subject makes either a guess response (with probability 1-q) or a SCR (with probability $q$ ). If the subject guesses, he makes response $A_{i}$ with (bias) probability $b_{i}$ regardless of which stimulus was presented. The latency of each guess response is assumed to be a random sample of a random variable $L_{g}$ with distribution function $\Phi_{g}$ [1.e., $\left.P\left(L_{g} \leq t\right)=\Phi_{g}(t)\right]$ and mean $\mu_{g}$. If the subject makes a $\mathrm{SCR}$, the response is correct with probability a>.5, and its latency is a random variable $L_{g}$ with distribution function $\Phi_{\mathrm{S}}$ and mean $\mu_{\mathrm{g}}$. The latency of a SCR is independent of which stimulus-response pair actually occurs. It is natural to suppose that guesses are faster than SCRs, i.e., that $\mu_{\mathrm{g}}<\mu_{\mathbf{g}}$, but the results given below do not depend on such an assumption.

In relating the model to observable quantities, the following notation is used: $p_{c}$ denotes the (marginal) probability of a correct response, $p_{e}$ the probability of an error, $F_{C}(t)$ the probability that a correct response latency is less than or equal to $t, F_{e}(t)$ the probability that an incorrect response latency is $\leq t, M_{C}$ the mean latency on correct responses, and $M_{e}$ the mean latency on incorrect responses. The following results can then be derived from the model:

$$
\begin{aligned}
& 2 p_{c} F_{c}(t)=c_{1}(t)\left[2 p_{c}-1\right]+\Phi_{g}(t) \\
& 2 p_{e} F_{e}(t)=c_{2}(t)\left[2 p_{c}-1\right]+\Phi_{g}(t) \\
& \frac{p_{c} F_{c}(t)-p_{e} F_{e}(t)}{p_{c}-p_{e}}=\Phi_{S}(t)
\end{aligned}
$$

where

$$
\begin{aligned}
& c_{1}(t)=\frac{2 a \Phi_{g}(t)-\Phi_{g}(t)}{2 \mathrm{a}-1} \\
& c_{2}(t)=\frac{2(1-\mathrm{a}) \Phi_{\mathrm{g}}(t)-\Phi_{g}(t)}{2 \mathrm{a}-1}
\end{aligned}
$$

Equation (3) is the correction for guessing formula that permits estimation of $\Phi_{s}$ from results of a single session. The following equations are immediate corollaries of (1), (2), and (3), respectively.

$$
\begin{aligned}
& 2 p_{c} M_{c}=c_{1}\left(2 p_{c}-1\right)+\mu_{g} \\
& 2 p_{e} M_{e}=c_{2}\left(2 p_{c}-1\right)+\mu_{g} \\
& \frac{p_{c} M_{c}-p_{e} M_{e}}{p_{c}-p_{e}}=H_{s}
\end{aligned}
$$

where $\quad c_{1}=\frac{2 a \mu_{s}-\mu_{g}}{2 a-1}, \quad c_{2}=\frac{2(1-a) \mu_{s}-\mu_{g}}{2 a-1}$

Equations (4) and (5) appear in Ollman's paper (in somewhat different notation); they describe linear relationships useful in testing the model. However, the test implied by (4) and (5) could fail if the latency distribution of guess responses varied from session to session. i.e., if $\mu \mathrm{g}$ varied. In such a case the model would remain essentially valid, but a plot of $2 \mathrm{p}_{c} \mathrm{M}_{\mathrm{c}}$ and $2 \mathrm{p}_{\mathrm{e}} \mathrm{M}_{\mathrm{e}}$ against $2 \mathrm{p}_{\mathrm{c}}-1$ would not reflect this. Consequently, it is of interest to observe that Equation (6) is independent of $\mu \mathrm{g}$, and thus provides a test of the Invariance of $\mu_{\mathbf{s}}$ that is unaffected by changes in guessing latencies, as well as guessing probabilities. Note that (6) can be obtained by subtracting (5) from (4).

Ollman's paper reports a test of the prediction that $2 \mathrm{p}_{\mathrm{c}} \mathrm{M}_{\mathrm{c}}$ and $2 \mathrm{pe}_{\mathrm{e}} \mathrm{M}_{\mathrm{e}}$ will be linearly related to $2 \mathrm{p}_{\mathrm{c}}-1$. A somewhat stronger test of the model is afforded by the fact that the relationship between $2 p_{c} M_{c}$ and $2 p_{c}-1$ can be predicted using the slope $\left(c_{2}\right)$ and intercept $\left(\mu_{g}\right)$ parameters estimated from a plot of $2 \mathrm{p}_{\mathrm{e}} \mathrm{M}_{\mathrm{e}}$ against $2 p_{c}-1$, the mean of the $\mu_{s}$ estimates provided by Equation (6), and the fact that $c_{1}-c_{2}=2 \mu_{\mathrm{s}}$. 


\section{Method}

Three hired subjects were run in a two stimulus-two response CRT experiment. There were 16 experimental sessions, each consisting of 480 trials. The choice stimulus on each trial was a red or green illumination of the screen of an IEE Series 10 read-out. In all sessions red and green stimuli occurred randomly over trials with equal probability. Responses were key depressions using the left or right forefinger. Within each session a fixed "deadline" condition prevailed; responses faster than the deadline were rewarded by computing the percentage of all such responses within each session and awarding one half cent for each percentage point. Immediate feedback on response speed was provided on each trial in the form of a $40 \mathrm{msec} 800 \mathrm{cps}$ tone burst that occurred if and only if the latency on that trial exceeded the prevailing deadline. Eight deadline conditions were studied: $150,200,250,300,350,400,500$, and $800 \mathrm{msec}$. Each condition was employed in two sessions, so that 960 responses were made by each subject under each deadline. Under all conditions subjects were
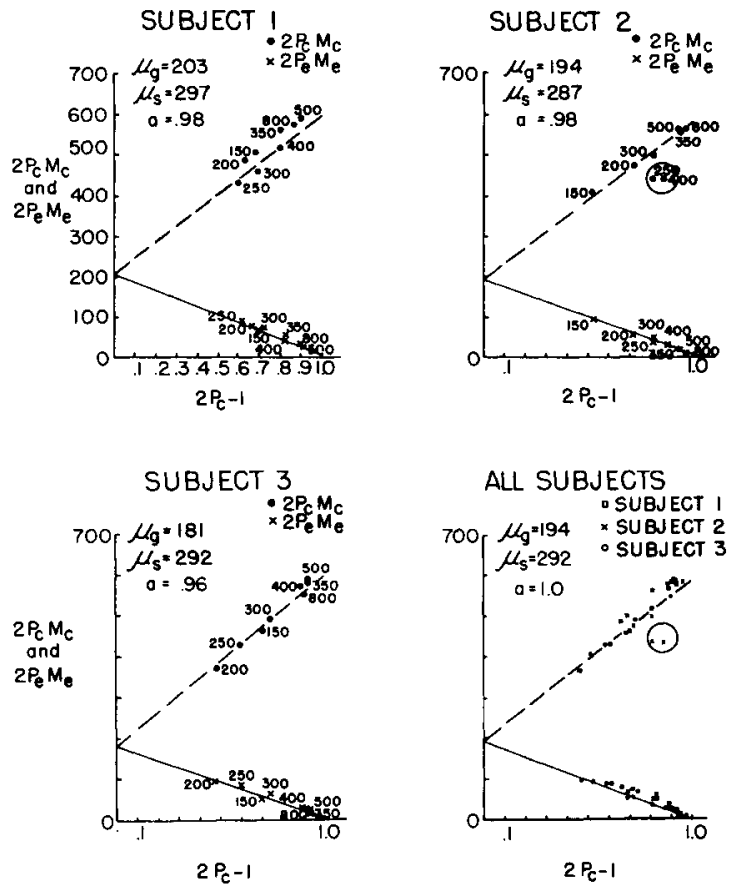

Fig. 1. Plots of $2 p_{c} M_{c}$ and $2 p_{e} M_{e}$ vs $2 p_{c^{-1}}$ for each $S$ and all Ss combined. Solid and dashed lines are theoretical lines based on Eqs. 4, 5, and 6. Numbers refer to deadline conditions. instructed to be "fast and accurate" and to try not to make more than 5\% errors. However, no penalty was imposed for errors. The three Ss had had extensive practice in earlier reaction time tasks, but were naive as to the purpose of the present experiment.

\section{Results}

For purposes of analysis the 960 responses made under each deadline condition were combined and treated as if they had been generated by a single session. Figure 1 shows the plots of $2 p_{c} M_{c}$ vs $2 p_{c}-1$, and $2 p_{e} M_{e}$ vs $2 p_{c}-1$, for each subject and for all subjects together. In each graph the lower line of points corresponds to $2 \mathrm{p}_{\mathrm{e}} \mathrm{M}_{\mathrm{e}}$, and the solid line through these points is the best fitting straight line as determined by least squares. The upper line of points corresponds to $2 \mathrm{p}_{\mathrm{c}} \mathrm{M}_{\mathrm{c}}$. The dashed line through these points is the relationship between $2 \mathrm{p}_{\mathrm{c}} \mathrm{M}_{\mathrm{c}}$ and $2 \mathrm{p}_{\mathrm{c}}-1$ predicted by using Equation (4), the least squares slope and intercept parameters of the $2 \mathrm{p}_{\mathrm{e}} \mathrm{M}_{\mathrm{e}}$ line, and an overall estimate of $\mu_{\mathrm{s}}$ obtained by averaging the $\mu_{\mathrm{s}}$ estimates from the various deadline conditions. For Subjects 1 and 3 all eight $\mu_{s}$ estimates were averaged to obtain the overall estimate used in prediction. For Subject 2 the anomalous values obtained under the $250 \mathrm{msec}$ and $400 \mathrm{msec}$ deadlines (the circled dots) were omitted in averaging. Except for these two points the observed values of $2 \mathrm{p}_{\mathrm{c}} \mathrm{M}_{\mathrm{c}}$ are quite close to the predicted line for each of the three subjects. The graph showing the combined results for all subjects is included to indicate the very minimal individual differences found in this study. The predicted $2 \mathrm{p}_{c} \mathrm{M}_{\mathrm{C}}$ line here was computed by treating the 24 data points as if they had been generated by a single subject and using the methods of estimation described above. To a good first approximation it appears that all three subjects were performing as predicted by the model, with mean reaction times of $190 \mathrm{msec}$ on guesses and 290 msec on stimulus controlled responses, and with essentially zero probability of an incorrect stimulus controlled response. This last is consistent with the fact that $s_{1}$ and $s_{2}$ here were perfectly discriminable.

\section{Reference}

Ollman, R. Fast guesses in choice reaction time. Psychon. Sci. $1966,6,155-156$.

\section{Note}

1. This research was supported by the University of Minnesota Space Science Center and the Center for Research in Human Learning. I thank Joan Tonn, who ran the Ss, and Peter Van Gelder who assisted in setting up the experiment. 